\title{
BMJ Open Association of prehypertension and cardiovascular risk factor clustering in Inner Mongolia: a cross-sectional study
}

Guoju Li, ${ }^{1}$ Guanghong Guo, ${ }^{2}$ Wenrui Wang, ${ }^{3}$ Ke Wang, ${ }^{4}$ Hailing Wang, ${ }^{3}$ Fen Dong, ${ }^{5}$ Yonggang Qian, ${ }^{3}$ Haiying Gong, ${ }^{6}$ Guodong Xu, ${ }^{5}$ Yanlong Li, ${ }^{1}$ Li Pan, ${ }^{1}$ Biao Zhang, ${ }^{1}$ Guangliang Shan ${ }^{1}$

To cite: Li G, Guo G, Wang W, et al. Association of prehypertension and cardiovascular risk factor clustering in Inner Mongolia: a cross-sectional study. BMJ Open 2017;7:e015340. doi:10.1136/ bmjopen-2016-015340

$\mathrm{GL}$ and $\mathrm{GG}$ contributed equally.

Received 29 November 2016 Revised 19 April 2017 Accepted 4 May 2017

\section{CrossMark}

${ }^{1}$ Department of Epidemiology and Statistics, Institute of Basic Medical Sciences, Chinese Academy of Medical Sciences, School of Basic Medicine, Peking Union Medical College, Beijing, China

${ }^{2}$ Department of Clinical Biochemistry, Chinese PLA General Hospital, Beijing, China ${ }^{3}$ Inner Mongolia Center for Disease Control and Prevention, Hohhot, China

${ }^{4}$ Department of Obstetrics, Key Laboratory of 9 Birth Defects and Related Diseases of Women and Children (Sichuan University), Ministry of Education, West China 10 Second University Hospital, Sichuan University, Chengdu, China

${ }^{5}$ China-Japan Friendship Hospital, Beijing, China

${ }^{6}$ Fangshan District Center for Disease Control and Prevention, Beijing, China

Correspondence to Guangliang Shan; guangliang shan@163.com

\section{ABSTRACT}

Objectives To assess the clustering of cardiovascular disease (CVD) risk factors in Han and Mongolian adults with prehypertension or hypertension in Northern China. Methods We selected 3227 Han and Mongolian participants (20-80 years old) using a multistage cluster sampling method in 2014. The participants were interviewed by standard questionnaires and underwent anthropometric measurement and biochemical testing. Han and Mongolian participants were divided into optimal, prehypertension, and hypertension groups based on blood pressure. A multinomial logit analysis was performed to explore relationships between CVD risk factor clustering and prehypertension or hypertension, and the heterogeneity between Han and Mongolian was evaluated by the Cochran $Q$ test. The differences between the ethnic groups in the proportions of risk factors was tested with the $\chi^{2}$ test.

Results The clustering of two or three CVD risk factors in the prehypertension or hypertension groups was consistently higher than in the optimal group (Bonferroni, $\mathrm{p}<0.0167)$. The odds ratios $(\mathrm{ORs})$ of prehypertension and hypertension increased with the number of CVD risk factors $\left(p_{\text {trend }}<0.0001\right)$. In multivariate modelling the adjusted ORs of one, two, and $\geq 3$ CVD risk factors versus no risk factors was, respectively, 1.95, 2.25, and 2.28 in Han prehypertensive participants, and 1.73, 2.83 , and 3.69 in Mongolian prehypertensive participants. In addition, the adjusted ORs were $3.15,4.75$, and 6.49 in Han hypertensive participants, and 1.90, 5.29, and 8.13 in Mongolian hypertensive participants (all $p<0.05$ ). There was no significant heterogeneity between Han and Mongolian participants in the prehypertension or hypertension groups. The age-standardised prevalence of $\geq 3$ risk factors was $38.30 \%$ in Han men and $39.79 \%$ in Mongolian men. The rate was significantly lower in Han women than Mongolian women (9.18\% vs $14.55 \%$, $\mathrm{p}=0.002$ ).

Conclusions These findings showed clustering of CVD risk factors in prehypertensive Han and Mongolian adults, and showed prehypertension may be a useful target for intervention.

\section{INTRODUCTION}

Cardiovascular disease (CVD) is a major cause of death worldwide, accounting

\section{Strengths and limitations of this study}

- The present study was first designed to assess the clustering of CVD risk factors in prehypertensive and hypertensive Han and Mongolian adults in Inner Mongolia, China.

- The high quality study design and implementation with a high response rate, the use of trained interviewers, and checks by the people who trained the interviewers on the responses of the participants, improved the validity of our self-reported data.

- The unclear temporal relationships between CVD risk factors and hypertension are due to inherent weaknesses of cross-sectional studies, and have been heatedly debated in epidemiological studies.

- Furthermore, important confounding factors possibly associated with CVD, such as nutrition and physical activity, were not evaluated in the present study.

- The age-standardised prevalence of CVD risk factor clustering was not shown by optimal, prehypertension, and hypertension groups because of small sample sizes.

for $>17$ million deaths in 2013. ${ }^{1}$ Hypertension is one of the most important risk factors for developing CVD. ${ }^{23}$ In 2010, hypertension was the leading risk factor for global disease burden, and it was the major contributor to CVD mortality in East Asia, Southeast Asia, Central Asia, the Caribbean, North Africa, and the Middle East. ${ }^{3}$ The seventh report of the Joint National Committee on Prevention, Detection, Evaluation, and Treatment of High Blood Pressure (JNC7) defined prehypertension as a systolic blood pressure (SBP) of $120-139 \mathrm{mmHg}$ and/or a diastolic blood pressure (DBP) of $80-89 \mathrm{mmHg} .{ }^{4}$ Further understanding has shown that hypertension is a consecutive process, from optimal to high blood pressure (BP). Studies have demonstrated that individuals with prehypertension are at an increased risk for developing hypertension and GVD. ${ }^{5-8} \mathrm{~A}$ prospective cohort study conducted in China also showed that 
the population-attributable risk associated with prehypertension was $10.6 \%$ and $7.1 \%$ for CVD incidence and mortality, ${ }^{9}$ respectively. In addition, it has been shown that if prehypertension was eliminated, $15.9 \%$ of CVD, $14.6 \%$ of coronary heart disease, and $19.6 \%$ of stroke cases could be prevented. ${ }^{10}$ Current smoking, overweight or obesity, diabetes, and dyslipidaemia are well-established risk factors for CVD. ${ }^{11-13}$ It has been demonstrated that hypertension is associated with both increased blood lipid levels ${ }^{14-16}$ and diabetes. ${ }^{17-19}$ Among the defined traditional CVD risk factors, hypertension is not only the most conveniently measurable, but is often the most easily controllable. However, it is unclear to what extent there is clustering of major CVD risk factors with prehypertension or hypertension.

The Inner Mongolia Autonomous Region is in Northern China, and Han and Mongolian constitute approximately $96 \%$ of the total population. These two ethnic groups have different genetic backgrounds, cultures, customs, and food consumption patterns. Several studies have noted striking ethnic disparities in CVD risk factor clustering in China and overseas. ${ }^{20-22}$ The present study aims to assess the clustering of CVD risk factors in Han and Mongolian adults with prehypertension or hypertension, which may assist in creating preventative measures against CVD in this population.

\section{METHODS}

\section{Study population}

A cross-sectional survey was performed in Bayan Nur, Xilingol League, Ulanqab, and Hohhot in Inner Mongolia, China, in 2014. The survey is one part of the China National Health Survey (CNHS), which is an ongoing national programme aiming to evaluate the 'physiological constant and health condition' in Chinese people. A sample of adults, aged 20-80 years old, was selected using a multistage cluster sampling method, which has been extensively described previously. ${ }^{23}$ Residents who had been living in Inner Mongolia for 1 year or longer were recruited, and all participants provided written informed consent. The survey was approved by the Institutional Review Board of the Institute of Basic Medical Sciences, Chinese Academy of Medical Sciences. In total, 3464 participants were investigated. Of these, 58 participants were excluded because of missing data, and 179 participants were excluded because their parents were not Mongolian or Han. Ultimately, a total of 3227 individuals were included in the analysis, including 2308 Han adults and 919 Mongolian adults.

\section{Health survey/measurements}

Data on demographic information, smoking, alcohol drinking, and history of diseases were collected with standard questionnaires. The use of trained interviewers, and checks by the people who trained the interviewers on the responses of the participants, improved the validity of the self-reported data. The ethnicity of participants was determined by their ID cards and their parents' ethnic status. Participants and their parents were required to be all Han or Mongolian adults. Alcohol consumption was divided into two categories: never-drinkers and ever-drinkers (including current drinkers and former drinkers). Information on personal history of hypertension and diabetes was also obtained. Height was measured to the nearest $0.1 \mathrm{~cm}$ using a fixed stadiometer, and weight was measured to the nearest $0.1 \mathrm{~kg}$ in a standing position by bioelectrical impendence analysis (BIA) with a commercially available body composition analyser (BC-420, TANITA, Japan) with participants in light clothes. Body mass index (BMI) was calculated as weight divided by height squared $\left(\mathrm{kg} / \mathrm{m}^{2}\right)$. Sitting BP was measured three times by trained research assistants following a standardised procedure using an Omron digital BP measuring device (Omron HEM-907, Japan). Blood samples were drawn after fasting overnight for at least 8 hours, and were immediately processed, refrigerated, and transported to the laboratory in Beijing. The blood samples were kept at $-80^{\circ} \mathrm{C}$ before biochemical testing. Fasting glucose and lipids, including total cholesterol (TC), triglycerides (TG), high-density lipoprotein cholesterol (HDL-C), and low-density lipoprotein cholesterol (LDL-C), were assessed in the General Hospital of Chinese People's Liberation Army (PLA).

\section{Definitions}

BP was divided into optimal BP, prehypertension, and hypertension. Optimal BP was defined as an average $\mathrm{SBP}<120 \mathrm{mmHg}$ and DBP $<80 \mathrm{mmHg}$. Prehypertension was defined as an average SBP of $120-139 \mathrm{mmHg}$ and/ or DBP of $80-89 \mathrm{mmHg}$. ${ }^{4}$ Hypertension was defined as an average $\mathrm{SBP} \geq 140 \mathrm{mmHg}$ and/or DBP $\geq 90 \mathrm{mmHg}$, or self-reported diagnosis of hypertension. ${ }^{24}$

BMI was graded into healthy (BMI $<25 \mathrm{~kg} / \mathrm{m}^{2}$ ), overweight (BMI $25-29 \mathrm{~kg} / \mathrm{m}^{2}$ ), and obese (BMI $\geq 30 \mathrm{~kg} / \mathrm{m}^{2}$ ) according to the WHO criteria. ${ }^{25}$ Diabetes was defined as fasting blood glucose (FPG) $\geq 7.0 \mathrm{mmol} / \mathrm{L}$ and $/$ or a previous diagnosis of diabetes, ${ }^{26}$ and dyslipidaemia was defined as $\mathrm{TC} \geq 6.22 \mathrm{mmol} / \mathrm{L}$ and $/$ or $\mathrm{TG} \geq 2.26 \mathrm{mmol} / \mathrm{L}$ and/or HDL-C $<1.04 \mathrm{mmol} / \mathrm{L}$ and/or LDL-C $\geq 4.14 \mathrm{mmol} / \mathrm{L} .{ }^{27}$ Current smoking status was determined when participants answered 'yes' to the question 'Do you smoke now?' and had smoked $\geq 1$ cigarette per day for at least 6 months. ${ }^{28}$

\section{Statistical analysis}

The data were expressed as mean $\pm \mathrm{SD}$ for continuous variables and percentage and $95 \%$ CI. The $\chi^{2}$ test or one-way analyses of variance were used to compare characteristics of optimal BP, prehypertensive and hypertensive participants in each ethnic group (see table 1). The Bonferroni test was used for multiple comparisons. Variables with a skewed distribution (eg, TG levels) were log-transformed and their $95 \%$ CI were reported.

A multinomial logit analysis was performed to evaluate the relationship between CVD risk factors (current 


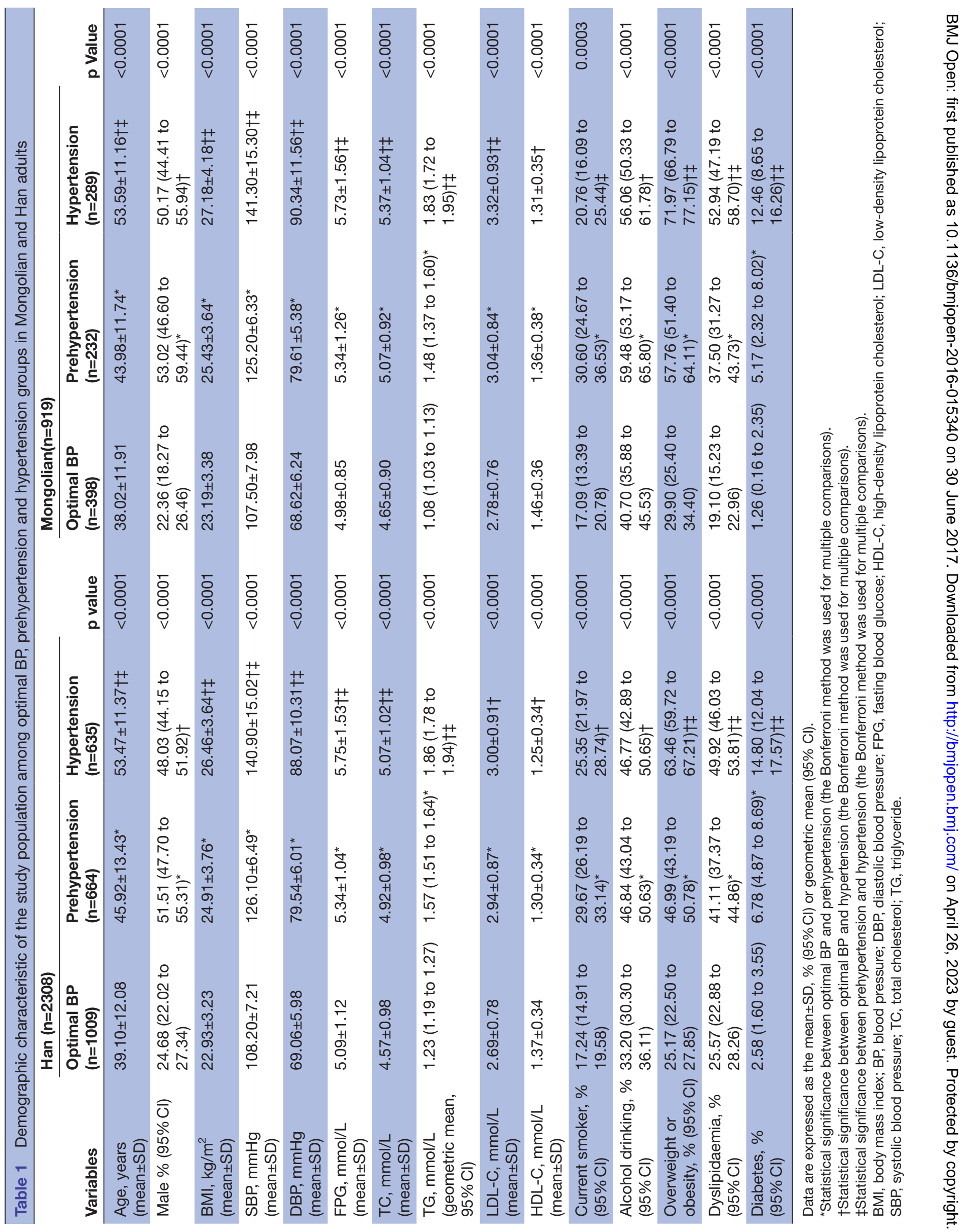


Table 2 Relationships of prehypertension and hypertension with CVD risk factors

\begin{tabular}{|c|c|c|c|c|}
\hline \multirow[b]{3}{*}{ CVD risk factors } & \multicolumn{2}{|l|}{ Han } & \multicolumn{2}{|l|}{ Mongolian } \\
\hline & Prehypertension $(n=664)$ & Hypertension $(n=635)$ & Prehypertension $(n=232)$ & Hypertension $(n=289)$ \\
\hline & OR $(95 \% \mathrm{Cl})$ & OR (95\% Cl) & OR $(95 \% \mathrm{Cl})$ & OR (95\% Cl) \\
\hline $\begin{array}{l}\text { Current smoking (yes } \\
\text { vs no) }\end{array}$ & 0.79 (0.57 to 1.09$)$ & 0.70 (0.49 to 1.01$)$ & 0.76 (0.45 to 1.27$)$ & $0.54(0.31$ to 0.93$)$ * \\
\hline $\begin{array}{l}\text { Overweight/obesity } \\
\text { (yes vs no) }\end{array}$ & $2.13(1.70$ to 2.67$)$ * & $3.82(3.00$ to 4.88$)$ * & 2.15 (1.49 to 3.12$)$ * & $3.09(2.07$ to 4.60$)$ * \\
\hline $\begin{array}{l}\text { Dyslipidaemia (yes vs } \\
\text { no) }\end{array}$ & 1.15 (0.91 to 1.46$)$ & $1.37(1.07$ to 1.76$)$ * & $1.53(1.02$ to 2.30$)$ * & $2.58(1.71$ to 3.91$)$ * \\
\hline Diabetes (yes vs no) & 1.44 (0.86 to 2.42$)$ & 2.36 (1.45 to 3.85$)$ * & 1.56 (0.52 to 4.71$)$ & 2.41 (0.87 to 6.71$)$ \\
\hline
\end{tabular}

Adjusted for age, gender, and alcohol drinking in multivariate logistic regression model.

${ }^{*}$ Compared with optimal blood pressure: $p<0.05$; CVD risk factors were included in models with an enter method.

CVD, cardiovascular disease.

smoking, BMI $\geq 25 \mathrm{~kg} / \mathrm{m}^{2}$, dyslipidaemia, diabetes) and prehypertension and hypertension (see table 2). Proportions of individuals with CVD risk factors clustering in optimal, prehypertensive and hypertensive groups were presented as percentage and $95 \% \mathrm{CI}$ and were analysed with the $\chi^{2}$ partition (CSP) method; the differences between the ethnic groups in the proportions of risk factors was tested with the $\chi^{2}$ test (see table 3 ).

A multinomial logit analysis was performed to explore the relationships of prehypertension and hypertension with one, two and $\geq 3$ risk factors clustering after adjustment for age, gender, and alcohol drinking in Han and Mongolian adults (see table 4). In the analysis, stratified by Han and Mongolian participants, optimal BP, prehypertension, and hypertension served as three levels of the dependent variable, and age, gender, alcohol consumption, and the number of CVD risk factors clustering were independent variables. The heterogeneity of associations between the Han and Mongolian participants was evaluated by using the Cochran $Q$ test. Additionally, dose-response relationships between CVD risk factor clustering and prehypertension or hypertension were examined using the number of CVD risk factors as a continuous variable in multinomial logit models.

Age direct standardisation of CVD risk factors to the National 2010 Census was conducted in the Han and Mongolian adults. A two-tailed $\mathrm{p}<0.05$ was considered statistically significant. All statistical analyses were performed using SAS software version 9.3 (SAS Institute Inc, Cary, NC, USA).

Table 3 Comparisons of proportions (\%) of different risk factors clustering among optimal blood pressure, prehypertension and hypertension groups in Han and Mongolian

\section{Number of risk factors clustering}

\begin{tabular}{llll}
\hline 0 & 1 & 2 & $\geq 3$
\end{tabular}

\begin{tabular}{|c|c|c|c|c|}
\hline \multicolumn{5}{|l|}{ Han } \\
\hline Optimal $(n=1009)$ & 53.82 (50.74 to 56.89 ) & 27.25 (24.51 to 30.00$) \ddagger$ & 13.78 (11.65 to 15.90$)$ & 5.15 (3.79 to 6.52$)$ \\
\hline $\begin{array}{l}\text { Prehypertension } \\
(n=664)\end{array}$ & $26.20(22.86 \text { to } 29.55)^{\star}$ & 36.14 (32.49 to 39.80$)^{*}$ & 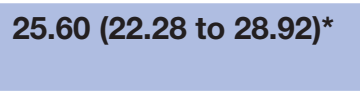 & $12.05(9.57 \text { to } 14.52)^{*}$ \\
\hline $\begin{array}{l}\text { Hypertension } \\
(\mathrm{n}=635)\end{array}$ & $14.65(11.90 \text { to } 17.40)^{\star} \dagger$ & $36.54(32.79 \text { to } 40.28)^{\star}$ & $31.50(27.88 \text { to } 35.11)^{\star} \rrbracket$ & $17.32(14.38 \text { to } 20.27)^{\star} \dagger$ \\
\hline \multicolumn{5}{|l|}{ Mongolian } \\
\hline Optimal $(n=398)$ & 50.75 (45.84 to 55.67 ) & 34.92 (30.24 to 39.61$)$ & 10.80 (7.75 to 13.85$)$ & 3.52 (1.71 to 5.33$)$ \\
\hline $\begin{array}{l}\text { Prehypertension } \\
(n=232)\end{array}$ & $21.55(16.26 \text { to } 26.84)^{\star}$ & 38.79 (32.52 to 45.06$)$ & 27.59 (21.83 to 33.34$)^{\star}$ & $12.07(7.88 \text { to } 16.26)^{\star}$ \\
\hline $\begin{array}{l}\text { Hypertension } \\
(\mathrm{n}=289)\end{array}$ & $13.15(9.25 \text { to } 17.04)^{\star} \dagger$ & $32.18(26.79$ to 37.57$)$ & $39.10(33.47 \text { to } 44.73)^{\star} \dagger$ & $15.57(11.39 \text { to } 19.75)^{\star}$ \\
\hline
\end{tabular}

${ }^{*}$ Compared with optimal blood pressure $p<0.0167$.

†Compared with pre-hypertension $p<0.0167$.

$\ddagger$ Compared with Mongolian $\mathrm{p}<0.05$ in optimal blood pressure.

ๆCompared with Mongolian $\mathrm{p}<0.05$ in hypertension. 
Table 4 Adjusted odds ratios of prehypertension and hypertension with CVD risk factor clustering

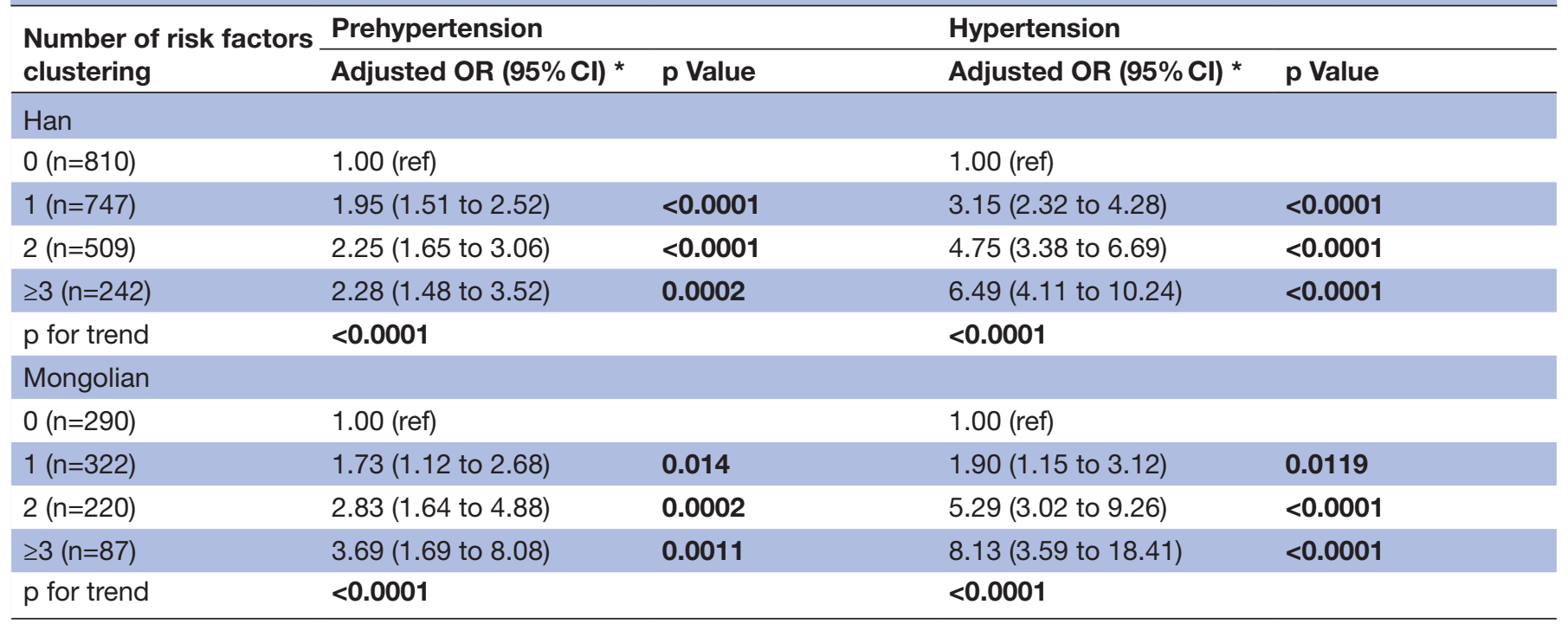

*The odds ratios were adjusted for age, gender and alcohol drinking in multinomial logistic regression models. CVD, cardiovascular disease.

\section{RESULTS}

A total of 3227 individuals were included in the analysis, including $2308(71.52 \%)$ Han adults and $919(28.48 \%)$ Mongolian adults. When compared with Han adults, the Mongolian adults were more likely to drink alcohol $(50.27 \%$ vs $40.86 \%)$, be overweight or obese $(50.16 \%$ vs $41.98 \%$ ), and have higher BMI (25.01 vs $24.47 \mathrm{~kg} / \mathrm{m}^{2}$ ), DBP (78.22 vs $77.31 \mathrm{mmHg})$, TC (4.98 vs $4.81 \mathrm{mmol} / \mathrm{L})$, LDL-C (3.02 vs $2.85 \mathrm{mmol} / \mathrm{L})$, and HDL-C (1.39 vs $1.32 \mathrm{mmol} / \mathrm{L}$ ) values (all $\mathrm{p}<0.05$ ). The prevalence of prehypertension and hypertension was $28.77 \%$ and $27.51 \%$ in the Han participants, and $25.24 \%$ and $31.45 \%$ in the Mongolian participants, respectively.

The characteristics of Han adults were similar to those of Mongolian adults. Specifically, the mean age was higher in the hypertension group than in the prehypertension and optimal BP groups (Bonferroni, all $\mathrm{p}<0.0167$ ) in both the Han and Mongolian participants. In each ethnic group, the mean BMI, SBP, DBP, fasting glucose, TC, and TG were consistently higher in participants with hypertension than in those with prehypertension (Bonferroni, all $\mathrm{p}<0.0167)$. These variables were also higher in the prehypertension group than in the optimal BP group (Bonferroni, all $\mathrm{p}<0.0167)$. HDL-C levels were lower in the optimal BP group than in the hypertension or prehypertension groups, and there was no statistical difference in values between the hypertension and prehypertension groups. The proportions of men in the prehypertension and hypertension groups were considerably higher than in the optimal BP group (Bonferroni, all $\mathrm{p}<0.0167$ ). Compared with the prehypertension and optimal groups, the proportions of overweight/obesity, dyslipidaemia, and diabetes were highest in the hypertension group (Bonferroni, $\mathrm{p}<0.0167$ ). Alcohol consumption differed only between the optimal BP group and either the prehypertension or hypertension group, without a statistical difference between the prehypertension and hypertension groups. In addition, ethnic disparities of smoking status were observed. The proportion of current smokers was higher in the hypertension and prehypertension groups than in the optimal BP group in the Han participants, while the proportion of current smokers in the prehypertension group was higher than in the optimal BP and hypertension groups in the Mongolian participants (Bonferroni, all $\mathrm{p}<0.0167$ ) (table 1).

A multinomial logit analysis was performed to evaluate the relationship between CVD risk factors and prehypertension or hypertension. Overweight or obesity was significantly associated with an increased risk of prehypertension and hypertension in both the Mongolian and Han adults (OR 2.13, 95\% CI 1.70 to 2.67 for prehypertensive Han; OR 3.82, 95\% CI 3.00 to 4.88 for hypertensive Han; OR 2.15, 95\% CI 1.49 to 3.12 for prehypertensive Mongolian participants; OR 3.09, 95\% CI 2.07 to 4.60 for hypertensive Mongolian participants, all $\mathrm{p}<0.05)$. Dyslipidaemia was significantly associated with an increased risk of prehypertension and hypertension in the Mongolian participants, but was only associated with hypertension in the Han participants $(\mathrm{p}<0.05)$. In the Han participants, those with diabetes experienced an increased risk of hypertension $(p<0.05)$. In the Mongolian participants, current smoking was significantly associated with a decreased risk of hypertension $(\mathrm{p}<0.05)$ (table 2$)$.

Overall, the proportion of participants with no CVD risk factors was $35.10 \%$ in the Han participants and $31.56 \%$ in the Mongolian participants, without a significant difference $(p>0.05)$. table 3 shows comparisons of CVD risk factor clustering among the optimal BP, prehypertension, and hypertension groups in Han and Mongolian participants. In each ethnic group, the proportions of two or $\geq 3$ CVD risk factor clustering were consistently higher in hypertensive or prehypertensive individuals 
than in those with optimal BP (Bonferroni, $\mathrm{p}<0.0167)$. Additionally, we observed that more hypertensive Han individuals had $\geq 3 \mathrm{CVD}$ risk factor clustering, compared with prehypertensive Han participants. In Mongolian participants, however, more hypertensive individuals had two CVD risk factor clustering than prehypertensive participants (Bonferroni, all $\mathrm{p}<0.0167$ ). There was no statistically significant difference between prehypertension and hypertension in the Mongolian participants with $\geq 3$ CVD risk factors. The proportions of one CVD risk factor clustering in the optimal BP group $(p=0.0045)$ and two CVD risk factors clustering in the hypertension group $(p=0.0236)$ were higher in the Mongolian adults than in the Han adults.

With the optimal BP group as a reference, the adjusted odds ratios (ORs) of prehypertension and hypertension associated with CVD risk factor clustering in Mongolian and Han participants were estimated in multinomial logistic models (table 4). In both Han and Mongolian adults, the ORs of prehypertension or hypertension increased with an increased number of CVD risk factor clustering $\left(\mathrm{p}_{\text {trend }}<0.0001\right)$. Compared with no risk factors, the adjusted ORs of prehypertension among one, two, and $\geq 3$ CVD risk factors were $1.95,2.25$, and 2.28 for the Han participants, and 1.73, 2.83, and 3.69 for the Mongolian participants, respectively (all $\mathrm{p}<0.05$ ). The adjusted ORs of hypertension among one, two, and $\geq 3 \mathrm{CVD}$ risk factors were $3.15,4.75$, and 6.49 for the Han participants, and $1.90,5.29$, and 8.13 for the Mongolian participants, respectively (all $\mathrm{p}<0.05)$. In the prehypertension group, there was no significant heterogeneity between the Han and Mongolian participants, and there was no heterogeneity in the hypertension group (both $\mathrm{p}>0.05$ ).

As hypertension is a well-known risk factor for CVD, we combined hypertension with the CVD risk factors mentioned above to assess the overall prevalence of CVD risk factor clustering. In men, the prevalences of one, two, and $\geq 3$ CVD risk factors were $23.03 \%, 28.90 \%$, and $38.30 \%$ in the Han participants, and $27.51 \%, 26.19 \%$, and $39.79 \%$ in the Mongolian participants, respectively. In women, the prevalences of one, two, and $\geq 3$ CVD risk factors were $26.36 \%, 18.29 \%$, and $9.18 \%$ in the Han participants, and $29.90 \%, 15.07 \%$, and $14.55 \%$ in the Mongolian participants, respectively. With respect to gender, men tended to have a higher prevalence of either two or $\geq 3$ CVD risk factor clustering than women $(p<0.05)$. The age-standardised prevalence of $\geq 3$ risk factor clustering was significantly lower in Han women than Mongolian women $(\mathrm{p}=0.002)$ (figure 1$)$.

\section{DISCUSSION}

Our study found that participants with prehypertension had higher levels of CVD risk factors such as BMI,

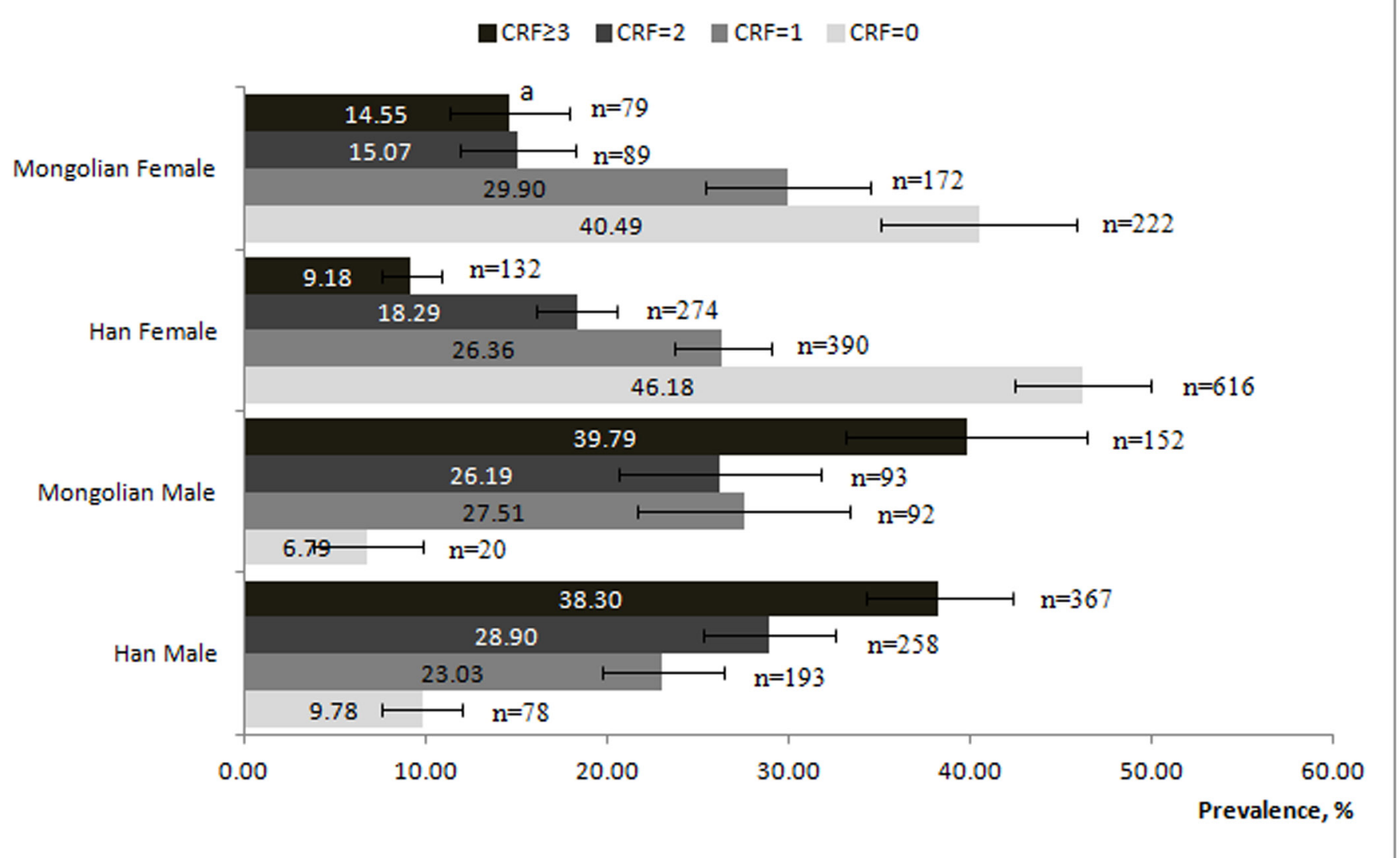

Figure 1 Age-standardised prevalence of major cardiovascular disease risk factors in Han and Mongolian adults by gender (\%). (a) The difference in the age-standardised prevalence of $\geq 3$ risk factor clustering between Han women and Mongolian women $(p=0.002)$. CRF, cardiovascular disease risk factor. 
FPG, TC, TG, and LDL-C than those with optimal BP, and that participants with hypertension had higher levels of BMI, FPG, TC, and TG than those with prehypertension. These findings indicate that hypertension is a consecutive process from optimal to high BP, and that prehypertension is an important intermediate phase before hypertension. A cohort study conducted in Brazil showed that four in five individuals with prehypertension, aged 40 to 49 years old, would be fully hypertensive within 10 years. ${ }^{29}$ Winegarden also reported that, compared with optimal BP, the estimated RR for the subcategory 'normal' (SBP 120-129 mmHg or DBP $80-85 \mathrm{mmHg}$ ) was 2.0 (95\% CI 1.6 to 2.6), and for 'high normal' values (SBP $130-139 \mathrm{mmHg}$ or DBP $85-89 \mathrm{mmHg}$ ) was 2.9 (95\% CI 2.3 to 3.7$).{ }^{30}$ In addition, the proportions of two and $\geq 3 \mathrm{CVD}$ risk factor clustering were higher in the prehypertension or hypertension groups than the optimal BP group. These results are consistent with the study conducted in Beijing in 2007, ${ }^{31}$ which suggested that CVD risk factor clustering was common among people with prehypertension and hypertension. Furthermore, our study showed a doseresponse relationship between the number of CVD risk factors and prehypertension or hypertension. Specifically, the ORs of prehypertension or hypertension increased as the number of CVD risk factors increased. A cohort study of 4.1 million adults in the UK showed that a $20 \mathrm{mmHg}$ higher SBP and a $10 \mathrm{mmHg}$ higher DBP were associated with a $58 \%$ and $52 \%$ higher risk of new-onset diabetes, respectively. ${ }^{32}$ A recent Korean epidemiology study also demonstrated that individuals with prehypertension (HR $1.27,95 \%$ CI 1.09 to 1.48 ) and hypertension (HR 1.51, $95 \%$ CI 1.29 to 1.76 ) had a higher risk of developing diabetes than those with optimal BP. ${ }^{33}$ A careful re-analysis of diagnosis times for hypertension and diabetes $(n=76)$ in our study showed that $74.67 \%(57 / 76)$ of hypertension diagnoses were made before diabetes diagnoses. Evidence in our study indicates that hypertension might be more likely to develop before diabetes. The prehypertensive participants had higher levels of TC, LDL-C, and TG, and lower levels of HDL-C than the optimal BP participants. ${ }^{34}$ Our findings also suggest that prehypertension may result in CVD risk factor clustering. As a modifiable condition, prehypertension may be a useful target for intervention, and antihypertensive medications have been found to reduce the relative risk of CVD and death by $15 \%$ in secondary prevention studies of prehypertension. ${ }^{35}$ In China, according to the national basic public health service specification, primary care practitioners should screen for hypertension in adults $\geq 35$ years old. Education of the public about the risks of prehypertension is a new challenge in primary care. In the JNC7 guidelines, the recommended management approach to uncomplicated prehypertension is health-promoting lifestyle modifications and antihypertensive medications given to adults with comorbid prehypertension and clinical cardiovascular disease ${ }^{36}$ Primary care practitioners must conduct further screening for prehypertension and prevent the incidence of hypertension, diabetes, and dyslipidaemia in their patients. Overweight or obesity has been linked to increased incidence of hypertension. ${ }^{37}$ In addition, BMI has also been shown to be an important risk factor for developing CVD. ${ }^{38}$ The association between smoking and hypertension is a more complex issue. Several studies have concluded that smokers have a lower prevalence of hypertension, ${ }^{39}$ while other studies have suggested that smoking is positively associated with hypertension. ${ }^{40}$ Despite the contradictory results, smoking is still a wellknown risk factor for developing CVD. ${ }^{41}$ Overall, body weight reduction, tobacco cessation, and $\mathrm{BP}$ control in prehypertensive patients are important ways to prevent hypertension, diabetes, and dyslipidaemia, thus lowering their risk of developing CVD.

When evaluating ethnic differences in CVD risk factors, we found that the Mongolian participants were more likely to have a lipid disorder and be overweight or obese than the Han participants. They also had higher fasting glucose levels than the Han participants. Such ethnic differences have been reported in previous studies. ${ }^{42} 43$ A cross-sectional study conducted in Xinjiang concluded that Mongolian adults $>30$ years old tend to have higher TC, LDL-C, FBG, and BMI than Han adults. ${ }^{20}$ Compared with a similar study in Inner Mongolia in 2003, ${ }^{44}$ the levels of TC, TG, HDL-C, LDL-C, FBG, and BMI of Mongolian adults in our study were higher, which may be due to economic development and lifestyle changes. ${ }^{45}$ When analysing ethnic differences in CVD risk factor clustering, we found that Mongolian participants with optimal BP had a higher proportion of one risk factor clustering than Han participants, and hypertensive Mongolian participants had a higher proportion of two risk factor clustering than hypertensive Han participants. This may be due to a different culture, customs, and food consumption patterns between the two ethnic groups. Traditionally, Mongolians are accustomed to a higher intake of animal fat and drink strong wine. A careful re-analysis of the participants' characteristics in our study demonstrated that the Mongolian participants had a higher proportion of overweight or obese participants $(71.97 \%$ and $63.46 \%$ in hypertension, respectively) than the Han participants, and they tended to drink more alcohol $(56.06 \%$ and $46.77 \%$ in hypertension, respectively). Differences in the prevalence of CVD risk factors between Han and Mongolian adults imply a need to develop tailored prevention programmes targeting ethnic groups, thereby reducing the prevalence of CVD risk factors in the general population.

Another important finding was the age-standardised prevalence of one, two, and $\geq 3$ CVD risk factor clustering in Mongolian and Han populations. About $90.22 \%$ of Han men and $93.21 \%$ of Mongolian men had at least one CVD risk factor, which was similar to the study conducted in Beijing in 2007 (91.3\%). ${ }^{46}$ However, the proportions of $\geq 3$ CVD risk factors in Han and Mongolian men were $38.30 \%$ and $39.79 \%$, respectively, which were higher than the results of the latter study $(22.6 \%) .{ }^{46}$ Because of the sharp differences in smoking rates $(55.58 \%$ in Han men 
vs $2.41 \%$ in Han women, $48.46 \%$ in Mongolian men vs $4.63 \%$ in Mongolian women), men had a much higher prevalence of CVD risk factor clustering than women. The age-standardised prevalence of the clustering of $\geq 3$ risk factors in Han women was significantly lower than that in Mongolian women. These findings indicate that men and Mongolian women should be the targeted population for CVD prevention and control.

Our study's limitations were mainly the unclear temporal relationships between CVD risk factors and hypertension, which is due to the inherent weakness of cross-sectional studies, and has been heatedly debated in epidemiological studies. A prospective cohort study needs to be completed to further assess the direction of prehypertension, hypertension, and CVD risk factors in Inner Mongolia. Furthermore, several important confounding factors associated with CVD, such as nutrition and physical activity, were not evaluated in the present study. Moreover, because of small sample sizes, the age-standardised prevalence of CVD risk factor clustering was not displayed by optimal BP, prehypertension, and hypertension groupings. Finally, this study was conducted only in Inner Mongolia, so the study's findings cannot be representative of all Chinese adults, and further research is needed.

In summary, several common CVD risk factors and their clustering were prevalent in the Han and Mongolian populations in Northern China. The clustering of CVD risk factors in prehypertensive patients suggests that prehypertension should be a key stage for early intervention of CVD. More strategies should also be developed, including screening of individuals with prehypertension and effective management of other common CVD risk factors. The gender disparities in CVD risk factor clustering suggest that targeted and cost-effective strategies for preventing CVD should be developed in Inner Mongolia.

Acknowledgements We sincerely express our gratitude to all the staff of the Inner Mongolian Autonomous Region Centre for Disease Control and Prevention for support with the collection of demographic data.

Contributors GL participated in the data collection and drafted the manuscript. $\mathrm{GG}, \mathrm{WW}, \mathrm{HW}, \mathrm{HG}, \mathrm{YQ}, \mathrm{GX}, \mathrm{YL}, \mathrm{BZ}$ participated in the data collection. KW, FD, LP, GS participated in the design of the study and undertook statistical analyses. All authors were involved in writing the paper and had final approval of the submitted and published versions.

Funding This study was supported by the National Science andTechnology Pillar Programme during the Twelfth Five-Year Plan Period sponsoredby the Ministry of Science and Technology of China (Grant No. 2012BAl37B02).

Competing interests None declared.

Patient consent Obtained.

Ethics approval Institutional Review Board of the Institute of Basic Medical Sciences, Chinese Academy of Medical Sciences (No.028-2013).

Provenance and peer review Not commissioned; externally peer reviewed.

Data sharing statement No additional data are available.

Open Access This is an Open Access article distributed in accordance with the Creative Commons Attribution Non Commercial (CC BY-NC 4.0) license, which permits others to distribute, remix, adapt, build upon this work non-commercially, and license their derivative works on different terms, provided the original work is properly cited and the use is non-commercial. See: http://creativecommons.org/ licenses/by-nc/4.0/

(c) Article author(s) (or their employer(s) unless otherwise stated in the text of the article) 2017. All rights reserved. No commercial use is permitted unless otherwise expressly granted.

\section{REFERENCES}

1. Global; GBD 2013 Mortality and Causes of Death Collaborators. Global, regional, and national age-sex specific all-cause and causespecific mortality for 240 causes of death, 1990-2013: a systematic analysis for the Global Burden of Disease Study 2013. Lancet 2015;385:117-71.

2. Mozaffarian D, Benjamin EJ, Go AS, As G, et al. Heart disease and stroke statistics--2015 update: a report from the American Heart Association. Circulation 2015;131:e29-322.

3. Lim SS, Vos T, Flaxman AD, et al. A comparative risk assessment of burden of disease and injury attributable to 67 risk factors and risk factor clusters in 21 regions, 1990-2010: a systematic analysis for the Global Burden of Disease Study 2010. Lancet 2012;380:2224-60.

4. Chobanian AV, Bakris GL, Black HR, et al. The Seventh Report of the Joint National Committee on Prevention, detection, evaluation, and treatment of high blood pressure: the JNC 7 report. JAMA 2003;289:2560-72.

5. Arima H, Murakami Y, Lam TH, et al. Effects of prehypertension and hypertension subtype on cardiovascular disease in the Asia-Pacific Region. Hypertension 2012;59:1118-23.

6. Fukuhara M, Arima H, Ninomiya T, et al. Impact of lower range of prehypertension on cardiovascular events in a general population: the Hisayama Study. J Hypertens 2012;30:893-900.

7. Assadi F. Prehypertension: a warning sign of future cardiovascular risk. Int J Prev Med 2014;5:S4-9.

8. Huang Y, Su L, Cai X, et al. Association of all-cause and cardiovascular mortality with prehypertension: a meta-analysis. Am Heart J 2014;167:160-8.

9. Gu D, Chen J, Wu X, et al. Prehypertension and risk of cardiovascular disease in Chinese adults. J Hypertens 2009;27:721-9.

10. Huang $Y$, Wang $S$, Cai $X$, et al. Prehypertension and incidence of cardiovascular disease: a meta-analysis. BMC Med 2013;11:177.

11. Ezzati M, Hoorn SV, Rodgers A, et al. Estimates of global and regional potential health gains from reducing multiple major risk factors. Lancet 2003;362:271-80.

12. Cooper RS, Orduñez P, Iraola Ferrer MD, et al. Cardiovascular disease and associated risk factors in Cuba: prospects for prevention and control. Am J Public Health 2006;96:94-101.

13. Yang ZJ, Liu J, Ge JP, Jp G, et al. Prevalence of cardiovascular disease risk factor in the Chinese population: the 2007-2008 China National Diabetes and Metabolic Disorders Study. Eur Heart $J$ 2012;33:213-20.

14. Freiberg JJ, Tybjaerg-Hansen A, Jensen JS, et al. Nonfasting triglycerides and risk of ischemic stroke in the general population. JAMA 2008;300:2142-52.

15. Ni WQ, Liu XL, Zhuo ZP, et al. Serum lipids and associated factors of dyslipidemia in the adult population in Shenzhen. Lipids Health Dis 2015;14:71.

16. Okamura T, Tanaka H, Miyamatsu N, et al. The relationship between serum total cholesterol and all-cause or cause-specific mortality in a 17.3-year study of a Japanese cohort. Atherosclerosis 2007;190:216-23.

17. Wilson PW, Meigs JB, Sullivan L, et al. Prediction of incident diabetes mellitus in middle-aged adults: the Framingham Offspring Study. Arch Intern Med 2007;167:1068-74.

18. Gress TW, Nieto FJ, Shahar E, et al. Hypertension and antihypertensive therapy as risk factors for type 2 diabetes mellitus. Atherosclerosis Risk in Communities Study. N Engl J Med 2000;342:905-12.

19. Golden SH, Wang NY, Klag MJ, et al. Blood pressure in young adulthood and the risk of type 2 diabetes in middle age. Diabetes Care 2003;26:1110-5.

20. Li N, Wang H, Yan Z, et al. Ethnic disparities in the clustering of risk factors for cardiovascular disease among the Kazakh, Uygur, Mongolian and Han populations of Xinjiang: a cross-sectional study. BMC Public Health 2012;12:499.

21. Gishti O, Gaillard R, Felix JF, et al. Early origins of ethnic disparities in cardiovascular risk factors. Prev Med 2015;76:84-91.

22. Bhopal RS, Humphry RW, Fischbacher CM. Changes in cardiovascular risk factors in relation to increasing ethnic inequalities in cardiovascular mortality: comparison of cross-sectional data 
in the Health Surveys for England 1999 and 2004. BMJ Open 2013;3:e003485.

23. Li G, Wang H, Wang K, et al. Prevalence, awareness, treatment, control and risk factors related to hypertension among urban adults in Inner Mongolia 2014: differences between Mongolian and Han populations. BMC Public Health 2016;16:294.

24. Egan BM, Zhao Y, Axon RN. US trends in prevalence, awareness, treatment, and control of hypertension, 1988-2008. JAMA 2010;303:2043-50.

25. Obesity: preventing and managing the global epidemic. Report of a WHO consultation. World Health Organ Tech Rep Ser 2000;894-1-253.

26. Alberti KG, Zimmet PZ, Definition ZPZ. Definition, diagnosis and classification of diabetes mellitus and its complications. Part 1: diagnosis and classification of diabetes mellitus provisional report of a WHO consultation. Diabet Med 1998;15:539-53.

27. Flink L, Underberg JA, Newman JD, et al. The recent national lipid association recommendations: how do they compare to other established dyslipidemia guidelines? Curr Atheroscler Rep 2015;17:494

28. Cosnes J, Carbonnel F, Beaugerie L, et al. Effects of cigarette smoking on the long-term course of Crohn's disease. Gastroenterology 1996;110:424-31.

29. Moreira LB, Fuchs SC, Wiehe M, et al. Incidence of hypertension in Porto Alegre, Brazil: a population-based study. $J$ Hum Hypertens 2008;22:48-50.

30. Winegarden CR. From "prehypertension" to hypertension? Additional evidence. Ann Epidemiol 2005;15:720-5.

31. Zhang WH, Zhang L, An WF, Wf A, et al. Prehypertension and clustering of cardiovascular risk factors among adults in suburban Beijing, China. J Epidemiol 2011;21:440-6.

32. Emdin CA, Anderson SG, Woodward M, et al. Usual blood pressure and risk of new-onset diabetes: evidence from 4.1 million adults and a meta-analysis of prospective studies. J Am Coll Cardiol 2015;66:1552-62.

33. Kim MJ, Lim NK, Choi SJ, et al. Hypertension is an independent risk factor for type 2 diabetes: the Korean genome and epidemiology study. Hypertens Res 2015;38:783-9.

34. Wu SL, Zhang ZQ, Song SB, et al. [Prevalence of prehypertension and associated cardiovascular risk: two years follow up results]. Zhonghua Xin Xue Guan Bing Za Zhi 2010;38:415-9.
35. Egan BM, Stevens-Fabry S. Prehypertension--prevalence, health risks, and management strategies. Nat Rev Cardiol 2015;12:289-300.

36. Chobanian AV, Bakris GL, Black HR, et al. Seventh report of the Joint National Committee on Prevention, detection, evaluation, and treatment of high blood pressure. Hypertension 2003;42:1206-52.

37. Thawornchaisit P, de Looze F, Reid CM, et al. Health risk factors and the incidence of hypertension: 4-year prospective findings from a national cohort of 60569 Thai Open University students. BMJ Open 2013;3:3.

38. Armstrong AC, Gidding SS, Colangelo LA, et al. Association of early adult modifiable cardiovascular risk factors with left atrial size over a 20-year follow-up period: the CARDIA study. BMJ Open 2014;4:e004001.

39. Gu D, Kelly TN, Wu X, et al. Mortality attributable to smoking in China. N Engl J Med 2009;360:150-9.

40. Halperin RO, Gaziano JM, Sesso HD. Smoking and the risk of incident hypertension in middle-aged and older men. $A m \mathrm{~J}$ Hypertens 2008;21:148-52.

41. Sipilä JO, Gunn JM, Kauko T, et al. Association of restaurant smoking ban and the incidence of acute myocardial infarction in Finland. BMJ Open 2016;6:e009320.

42. Walker SE, Gurka MJ, Oliver MN, et al. Racial/ethnic discrepancies in the metabolic syndrome begin in childhood and persist after adjustment for environmental factors. Nutrition, Metabolism and Cardiovascular Diseases 2012;22:141-8. Nutrition, metabolism, and cardiovascular diseases: NMCD.

43. Chiu M, Austin PC, Manuel DG, et al. Comparison of cardiovascular risk profiles among ethnic groups using population health surveys between 1996 and 2007. CMAJ 2010;182:E30 1-E310.

44. Li H, Xu T, Tong W, et al. Comparison of cardiovascular risk factors between prehypertension and hypertension in a Mongolian population, Inner Mongolia, China. Circ J 2008;72:1666-73.

45. Yusuf S, Reddy S, Ounpuu S, et al. Global burden of cardiovascular diseases: part I: general considerations, the epidemiologic transition, risk factors, and impact of urbanization. Circulation 2001;104:2746-53.

46. Zhang L, Qin LQ, Cui HY, et al. Prevalence of cardiovascular risk factors clustering among suburban residents in Beijing, China. Int $J$ Cardiol 2011;151:46-9. 